\title{
Coherent vertical electron transport and interface roughness effects in AIGaN/GaN intersubband devices
}

\author{
A. Grier, ${ }^{1, a)}$ A. Valavanis, ${ }^{1}$ C. Edmunds, ${ }^{2}$ J. Shao, ${ }^{2}$ J. D. Cooper, ${ }^{1}$ G. Gardner,,${ }^{3,4}$ \\ M. J. Manfra, ${ }^{2,3,4,5}$ O. Malis, ${ }^{2}$ D. Indjin, ${ }^{1}$ Z. Ikonić, ${ }^{1, b)}$ and P. Harrison ${ }^{6}$ \\ ${ }^{1}$ Institute of Microwaves and Photonics, School of Electronic and Electrical Engineering, \\ University of Leeds, Leeds LS2 9JT, United Kingdom \\ ${ }^{2}$ Department of Physics, Purdue University, West Lafayette, Indiana 47907, USA \\ ${ }^{3}$ Birck Nanotechnology Center, West Lafayette, Indiana 47907, USA \\ ${ }^{4}$ School of Materials Engineering, Purdue University, West Lafayette, Indiana 47907, USA \\ ${ }^{5}$ School of Electrical and Computer Engineering, Purdue University, West Lafayette, Indiana 47907, USA \\ ${ }^{6}$ Materials and Engineering Research Institute, Sheffield Hallam University, Sheffield S1 IWB, \\ United Kingdom
}

(Received 22 July 2015; accepted 20 November 2015; published online 11 December 2015)

\begin{abstract}
We investigate electron transport in epitaxially grown nitride-based resonant tunneling diodes (RTDs) and superlattice sequential tunneling devices. A density-matrix model is developed, and shown to reproduce the experimentally measured features of the current-voltage curves, with its dephasing terms calculated from semi-classical scattering rates. Lifetime broadening effects are shown to have a significant influence in the experimental data. Additionally, it is shown that the interface roughness geometry has a large effect on current magnitude, peak-to-valley ratios and misalignment features; in some cases eliminating negative differential resistance entirely in RTDs. Sequential tunneling device characteristics are dominated by a parasitic current that is most likely to be caused by dislocations; however, excellent agreement between the simulated and experimentally measured tunneling current magnitude and alignment bias is demonstrated. This analysis of the effects of scattering lifetimes, contact doping and growth quality on electron transport highlights critical optimization parameters for the development of III-nitride unipolar electronic and optoelectronic devices. (C) 2015 AIP Publishing LLC. [http://dx.doi.org/10.1063/1.4936962]
\end{abstract}

\section{INTRODUCTION}

Intersubband optoelectronic devices such as quantum cascade lasers (QCLs) and quantum-well infrared photodetectors (QWIPs) have predominantly been fabricated using lattice-matched $\mathrm{AlGaAs} / \mathrm{GaAs}$ or InGaAs/InAlAs heterostructures. Although a wide range of high-quality devices have been realized, these conventional materials present a number of intrinsic limitations. For example, terahertzfrequency $(\mathrm{THz}) \mathrm{QCLs}^{1}$ are the most powerful electrically driven compact sources of coherent radiation in the $1-5 \mathrm{THz}$ band, with numerous potential sensing and imaging applications in astronomy, pharmaceutical, and security scenarios. ${ }^{2}$ Peak $\mathrm{THz}$ emission powers in excess of $1 \mathrm{~W}$ (Ref. 3) are now available. However, the commercial impact of $\mathrm{THz}$ QCLs has been limited by the requirement for cryogenic cooling (currently $<200 \mathrm{~K}$ (Ref. 4)). Emission frequencies are also limited to $<5 \mathrm{THz},{ }^{5}$ principally by Reststrahlen absorption effects, owing to the relatively small $36 \mathrm{meV}$ longitudinal-optic (LO) phonon energy in GaAs, and this limits the range of potential spectroscopy applications of existing THz QCLs.

The AlGaN/GaN material system has been proposed as a highly promising alternative to conventional III-V systems including mid-infrared and THz QCLs ${ }^{2,6-8}$ and QWIPs. ${ }^{9}$ The higher LO-phonon energy $(92 \mathrm{meV})$ could potentially allow

\footnotetext{
${ }^{a)}$ Electronic mail: atgrier4@gmail.com

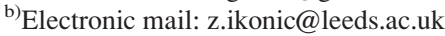

emission at higher $\mathrm{THz}$ frequencies, while the higher conduction band discontinuity $(1.75 \mathrm{eV}$ compared to $1 \mathrm{eV}$ in $\mathrm{AlGaAs} / \mathrm{GaAs}$ ) could reduce leakage currents, and therefore enable higher temperature operation. A detailed understanding of the carrier transport in $\mathrm{AlGaN} / \mathrm{GaN}$ heterostructures is critical to optimizing their performance and ultimately realizing high-quality optoelectronic devices. Resonant tunneling diodes (RTDs) are the simplest devices in which to explore vertical tunneling transport and they have undergone extensive experimental and theoretical investigation since the pioneering work by Esaki and Tsu. ${ }^{10}$ While they are well studied in arsenide ${ }^{11}$ and antimonide ${ }^{12}$ materials, measurement in nitrides remains relatively challenging. The existence of defects such as charge traps and screw dislocations has led to the need for systematic verification of the origin of negative differential resistance (NDR) features. ${ }^{13-17}$ Another important characteristic of $\mathrm{AlGaN} / \mathrm{GaN}$ heterostructures is the large built-in electrostatic fields due to both spontaneous and piezoelectric polarization which alter the current-voltage $(I-V)$ characteristics significantly. Recent advances in growth technology have reduced threading dislocation densities substantially to allow repeatable measurement of wurtzite and cubic AlGaN RTDs ${ }^{18-23}$ and sequential tunneling devices. $^{24,25}$ Furthermore, NDR features have also been demonstrated in defect-free nanowires. ${ }^{26-29}$ Intersubband absorption at both near-infrared $\mathrm{d}^{30-32}$ and $\mathrm{THz}^{33}$ wavelengths as well as mid-IR ${ }^{34}$ and $\mathrm{THz}^{35}$ electroluminescence has also been demonstrated, indicating that high-quality optoelectronic devices may soon be realized. 
Sequential tunneling devices rely on repeated tunneling and scattering of carriers through up to several hundred periods of a structure. It was first demonstrated in nitride devices by Sudradjat et al. ${ }^{36}$ with 20-30 three-well periods of an $\mathrm{Al}_{0.15} \mathrm{Ga}_{0.85} \mathrm{~N} / \mathrm{GaN}$ structure at low temperature with good agreement between the experimental and predicted subbandalignment voltages. Following this, a thinner structure with 10 periods of a single well and AlN barriers was grown and compared with analytical expressions ${ }^{25}$ for current; however, it was found that domain formation dominates the $I-V$ characteristics, preventing investigation into the roles of scattering on transport. To date, there has been no detailed theoretical study and comparison of devices which require scattering and tunneling between several states per period even though several exist for High Electron-Mobility Transistor (HEMT) structures. ${ }^{37,38}$

Several approaches exist for the modeling of RTD current-voltage characteristics including the transfer matrix, ${ }^{10}$ Wigner functions, ${ }^{39-41}$ and non-equilibrium Green's function (NEGF) methods. ${ }^{11}$ To date, nitride RTDs have been studied with the transfer matrix approach ${ }^{42}$ which assumes purely ballistic (coherent) transport through the double barrier structure, and also by the NEGF approach ${ }^{43}$ which is computationally intensive but describes scattering in the presence of coherent transport. Even fewer theoretical results are available for sequential tunneling transport due to its recent experimental realization.

In this work, we aim to unify transport modeling for RTDs and sequential tunneling devices by developing a modified form of the density matrix (DM) approach that uses relaxation terms calculated from all relevant scattering mechanisms. The DM approach has been well studied and shown to have good $I-V$ and output power agreement with experimental AlGaAs/GaAs QCLs. ${ }^{44}$ By comparing output from the model with high quality nitride experimental devices, we show the relative importance of coherent and incoherent transport mechanisms and the effect they have on critical characteristics such as the current peak-to-valley ratio, magnitude of current and high temperature behavior.

\section{RESONANT TUNNELING DIODES}

\section{A. Device fabrication and characterization}

Electrons in an RTD travel from a highly doped emitter region into a double barrier structure with resonant quantized subbands and then on to a collector region. By applying a bias to the device, the quantized states move in and out of alignment with a distribution of carriers in the emitter, causing NDR features in their $I-V$ characteristics.

$\mathrm{Al}_{0.18} \mathrm{Ga}_{0.82} \mathrm{~N} / \mathrm{GaN}$ RTDs with $49 \AA$ wells (barriers $24 \AA$ ) were grown using plasma-assisted molecular beam epitaxy (MBE) on high-quality free-standing $\mathrm{n}^{++} \mathrm{GaN}$ substrates, which were grown using hydride vapor phase epitaxy (HVPE) (dislocation density $<5 \times 10^{6} \mathrm{~cm}^{-2}$ ) and supplied by Kyma Technologies. ${ }^{19,20}$ Low $\mathrm{Al}$ composition was used to suppress relaxation effects of the strained $\mathrm{AlGaN}$ barrier layers during growth/processing and also to minimize electrical breakdown through interaction of the applied bias with polarization discontinuities. The emitter and collector regions consisted of $\mathrm{GaN}$ with silicon doping at a level of
$1 \times 10^{19} \mathrm{~cm}^{-3}$ separated by $20 \AA$ spacer layers from the well structure. After processing into $4 \times 4 \mu \mathrm{m}$ mesas, the chips were then mounted on copper blocks and wirebonded to gold contact pads before measurement in a liquid nitrogen-flow cryostat.

\section{B. Density-matrix model}

In our DM model, the device is split into three sections (the emitter, well, and collector) and it is assumed that the barriers are sufficiently thick or tall enough to limit transport to quantum tunneling only. This is appropriate since incoherent scattering will dominate transport within each section independently. We use the self-consistent Schrödinger-Poisson solver nextnano $3^{45}$ to calculate steady-state conduction band profiles which include the internal electric fields and the effects of contact Fermi level pinning and carrier distributions at each voltage step. To calculate the current characteristics for this system, we solve the Liouville equation

$$
\frac{\partial \rho}{\partial t}=-\frac{\mathrm{i}}{\hbar}[\mathrm{H}, \rho]-\frac{\rho}{\tau},
$$

which describes the evolution of the density terms in time. Localized wavefunctions are obtained in each of the three sections of the device, using an effective mass Schrödinger solver ${ }^{46}$ that accounts for non-parabolicity effects. These wavefunctions represent a "tight-binding" scheme where other sections of the device are replaced with barrier material. The resulting electron probability densities are shown in Fig. 1. These are then used as basis states for coherent transport through the device. The density matrix is expressed in block form as

$$
\rho=\left(\begin{array}{ccc}
\rho_{\mathrm{EE}} & \rho_{\mathrm{EW}} & \rho_{\mathrm{EC}} \\
\rho_{\mathrm{WE}} & \rho_{\mathrm{WW}} & \rho_{\mathrm{WC}} \\
\rho_{\mathrm{CE}} & \rho_{\mathrm{CW}} & \rho_{\mathrm{CC}}
\end{array}\right),
$$

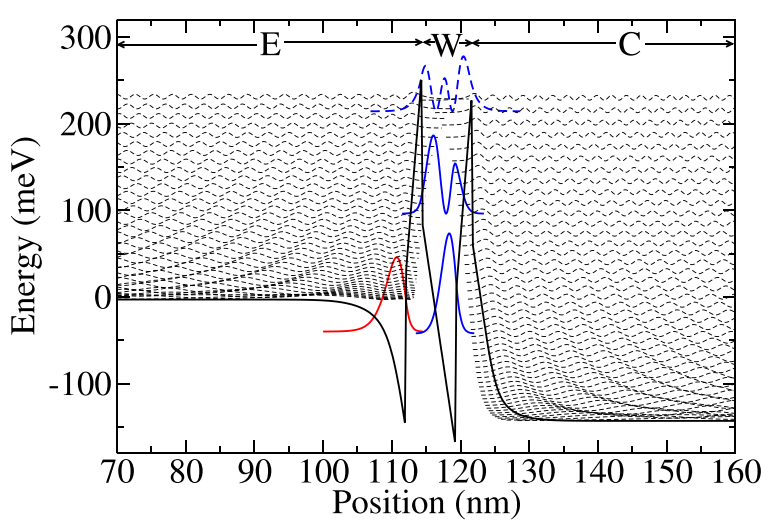

FIG. 1. Bandstructure and wavefunction plot of the $\mathrm{Al}_{0.18} \mathrm{Ga}_{0.82} \mathrm{~N} 49 \AA$ RTD at $0.136 \mathrm{~V}$. The localized wavefunctions are obtained using a "tight-binding" scheme with the device split into emitter (E), well (W), and collector (C) regions. The tight-binding Hamiltonian for the well section of the device is achieved by replacing the extended bandstructure in the emitter/collector with a potential equivalent to the maximum potential value of the barriers. The quantized emitter state and well confined states are shown in red and blue, respectively. 
where $\mathrm{E}, \mathrm{W}$, and $\mathrm{C}$ refer to emitter, well, and collector states, respectively. Combinations of these labels, such as EW, refer to any interaction involving states in the two specified regions and are used to describe the coupling strengths and dephasing times in addition to the coherence terms here. Each of the element-blocks within Eq. (2) is a sub-matrices, which represents the coherences of all pairs of states either within a given region (e.g., $\rho_{\mathrm{EE}}$ ) or between two different regions (e.g., $\rho_{\mathrm{EW}}$ ). In each of these blocks, the density terms are unknown values to be calculated and refer to the ensemble average of the weightings for the basis states $\rho_{i j}=\left\langle c_{i} c_{j}^{*}\right\rangle$. The physical interpretation of the diagonal $(i=j)$ elements is the probability of an electron being found in state $i$, and therefore the $i$ th subband populations can be determined by knowing the total carrier density. The off-diagonal elements represent the degree of polarization between states $i$ and $j$, which is interpreted as the coherence between the states. $\rho_{\mathrm{EC}}$ and its Hermitian adjoint are set to zero to indicate noninteraction between these sections. The emitter and collector reservoirs are set large enough to approximate a continuum of states such as those shown in Fig. 1. The size of the system is therefore $\left(N_{\mathrm{E}}+N_{\mathrm{W}}+N_{\mathrm{C}}\right)^{2}$, where $N$ is the number of states for each section. The Hamiltonian for the unperturbed system is

$$
\mathrm{H}=\left(\begin{array}{ccc}
H_{\mathrm{EE}} & H_{\mathrm{EW}} & 0 \\
H_{\mathrm{WE}} & H_{\mathrm{WW}} & H_{\mathrm{WC}} \\
0 & H_{\mathrm{CW}} & H_{\mathrm{CC}}
\end{array}\right)
$$

where the diagonal elements consist of the basis state energies. The off-diagonal elements within the intra-region blocks (EE, WW, and CC) are zero since no optical interaction is assumed. The inter-region blocks (EW, WE, WC, and $\mathrm{CW}$ ) describe the coupling between states and consist of the coupling strength (Rabi oscillation) terms calculated as ${ }^{47}$

$$
\hbar \Omega_{i j} \approx \sqrt{\left\langle i\left|\mathrm{H}_{\mathrm{ext}}-\mathrm{H}_{\mathrm{left}}\right| j\right\rangle} \times \sqrt{\left\langle i\left|\mathrm{H}_{\mathrm{ext}}-\mathrm{H}_{\mathrm{right}}\right| j\right\rangle},
$$

where $\mathrm{H}_{\text {ext }}$ and $\mathrm{H}_{\text {left,right }}$ refer to the Hamiltonians (potentials) of the extended structure and of the "tight-binding" sections, respectively. Several approaches are possible for the approximation of a tight-binding approach for resonant tunneling diodes; these are not as intuitive as the case for QCL or sequential tunneling structures as the majority of the contact regions are flat with most potential drop occurring over the well structure. Sections other than that being considered (emitter, well, or collector) are replaced with a potential corresponding to the maximum value of the potential in the device. These are replaced so that the internal electric fields are still accounted for (e.g., the potential substitution for the emitter region is performed after the first barrier).

If two energy levels of neighboring sections couple coherently, electron wave packets can propagate (tunnel) through the barrier from one energy level to another. The coherent transport depends on the strength of the coupling, the detuning from resonance, and the lifetime of the coherence.

Electron wave packets within each section of the device lose phase coherence due mainly to intrasubband elastic scattering, and several methods have been proposed for the approximation of dephasing times. We use an approach similar to that in Refs. 48 and 49 with the contributions from inter- and intrasubband scattering rates for the emitter and collector reservoirs. These calculations are performed for scattering due to LO phonons, acoustic phonons, alloy disorder, interface roughness, and ionized impurities. Dephasing due to intrasubband events in the well region are neglected as these are highly dependent on the well state populations which are not known in advance. By including intersubband scattering here, carriers can tunnel from the emitter to the excited state of the well, and proceed to scatter and tunnel to the collector from the well ground state.

The intrasubband electron-electron scattering rate was calculated to be approximately $W_{i i}=1 \times 10^{13} \mathrm{~s}^{-1}$ at $77 \mathrm{~K}$ and this was applied to all subbands to account for dephasing by this mechanism. Formally, the relaxation terms should obey the Lindblad master equation to ensure that the density matrix is positive definite. QCL simulations with a DM approach described here have reported negative populations at some in-plane wave vectors $k .{ }^{50}$ However, Fermi-Dirac averaged results retain positive populations and this was also observed in this work. To determine lifetimes, the total sheet-density of electrons (equal to the density of ionized impurities) was assumed to be distributed thermally across the continuum of states, with each subband electron temperature $\left(\mathrm{T}_{\mathrm{e}}\right)$ equal to the lattice temperature $\left(T_{\text {latt }}\right)$. The average scattering lifetimes $\tau_{i j}$ were then obtained by averaging over the in-plane wave vector of the initial state to include final state blocking as

$$
\frac{1}{\tau_{i j}}=\frac{\int W_{i j}\left(k_{i}\right) f_{i}^{\mathrm{FD}}\left(k_{i}\right)\left[1-f_{j}^{\mathrm{FD}}\left(k_{j}\right)\right] k_{i} \mathrm{~d} k_{i}}{\pi N_{i}},
$$

where $i$ and $f$ refer to the initial and final wavevector states for the scattering transition, $f_{i}^{\mathrm{FD}}$ is the Fermi-Dirac distribution for the $i$ th state, $N_{i}$ is the $2 \mathrm{D}$ sheet density, $k_{i}$ is the initial state wavevector, and $\hbar \omega$ is the transition energy (accounting for phonon interactions where applicable). Averaging in this way avoids the unbounded number of density matrices possible for the in-plane wavevector. Dephasing rates are then calculated from these states as ${ }^{48,49}$

$$
\frac{1}{\tau_{\| i, j}}=\frac{1}{2 \tau_{i}}+\frac{1}{2 \tau_{j}}+\frac{1}{\tau_{i i}}+\frac{1}{\tau_{j j}}-\frac{2}{\sqrt{\tau_{\mathrm{ifr}, i i} \times \tau_{\mathrm{ifr}, j j}}},
$$

where $\tau_{i}$ is the state lifetime calculated as $1 / \tau_{i}=\sum_{j} 1 / \tau_{i j}$, and $\tau_{i i}$ and $\tau_{\mathrm{ifr}, i i}$ are the total and interface roughness intrasubband scattering lifetimes, respectively. The scattering rates and dephasing times associated with all scattering rates except for interface roughness can be calculated a priori. However, interface roughness scattering rates exhibit a sample-specific dependence on the quality of epitaxial growth.

We use an interface-roughness (IFR) scattering model ${ }^{51}$ in which the roughness follows a Gaussian potential with r.m.s. height $\Delta$ and correlation length $\Lambda$. The scattering of carriers between states with an initial wavevector $k_{i}$ is given by 


$$
W_{i j}\left(k_{i}\right)=\frac{\pi m_{\mathrm{c}}}{\hbar^{3}} \Delta^{2} \Lambda^{2} \beta\left(k_{i}\right) \sum_{I}\left|V_{0} \Psi_{j}^{*}\left(z_{I}\right) \Psi_{i}\left(z_{I}\right)\right|^{2},
$$

where $m_{c}$ is the effective mass, and $\Psi_{i}$ and $\Psi_{j}$ are the wave functions of the initial and final states. $I$ is an index indicating each $\mathrm{AlGaN} / \mathrm{GaN}$ interface, $V_{0}$ is the step in conduction band potential at the interface and

$$
\beta\left(k_{i}\right)=\mathrm{e}^{-\left(k_{i}^{2}+k_{j}^{2}\right) \Lambda^{2} / 4} I_{0}\left(\frac{k_{i} k_{j} \Lambda^{2}}{2}\right) \Theta\left(k_{j}^{2}\right)\left[1-P_{j}\left(k_{j}\right)\right],
$$

where $I_{0}(\cdot)$ is the regular modified cylindrical Bessel function of zeroth order, $k_{i}$ and $k_{j}$ are the initial and final electron wavevectors, respectively, and $P_{j}$ is the occupation factor of the final wavevector. $\Theta(\cdot)$ is the Heaviside step function, which is used to ensure that transitions are energetically permissible. Eq. (7) indicates that the scattering rate (and therefore dephasing time) will depend on the parameters $\Delta$ and $\Lambda$ which are determined by the growth quality. The effect of varying these parameters is discussed in the results section. These rates are then used in the relaxation matrix

$$
\frac{\rho}{\tau}=\left(\begin{array}{ccc}
-\frac{\rho_{11}}{\tau_{1}}+\sum_{i \neq 1}^{N} \frac{\rho_{i i}}{\tau_{i 1}} & \cdots & \frac{\rho_{1 N}}{\tau_{\|, 1 N}} \\
\vdots & \ddots & \vdots \\
\frac{\rho_{N 1}}{\tau_{\|, N 1}} & \cdots & -\frac{\rho_{N N}}{\tau_{N}}+\sum_{i \neq N}^{N} \frac{\rho_{i i}}{\tau_{i N}}
\end{array}\right)
$$

which is the final term in Eq. (1). These relaxation matrix elements determine the duration of coherence between states, and therefore the magnitude of current and state broadening. For significant electron wave propagation between regions, the Rabi oscillation frequencies (related to coupling strength) must be faster than these dephasing terms.

\section{Steady state solution and current}

The Tsu-Esaki formalism for current assumes a Fermi-Dirac distribution of carriers in the reservoir regions with Fermi energies pinned to contacts on each side of the device to determine the magnitude of current. However, since the subband quasi-Fermi energy was set before the calculation of scattering rates, the solution for the diagonal $\rho$ elements naturally resembles a Fermi-Dirac distribution in each reservoir. Eq. (1) is solved with $\frac{\partial \rho}{\partial t}=0$ to find the steady-state emitter and well state populations and coherences using the Armadillo/LAPACK $\mathrm{C}++$ linear algebra libraries. ${ }^{52,53}$ To make the system inhomogeneous, trace conditions for the reservoirs were set so that $\sum_{i} \rho_{i i}=1$. Physical quantities such as current density for this device can be extracted from the solved density matrix as $j=\operatorname{Tr}(\rho J)$, with

$$
J=e \frac{\mathrm{i}}{\hbar}[\mathrm{H}, \mathrm{z}],
$$

where $e$ is unit charge and $\mathrm{z}$ consists of the dipole matrix element terms $z_{i, j}=\left\langle\Psi_{i}|z| \Psi_{j}\right\rangle$. It is worth noting that secondorder density matrix approaches have shown better agreement with experimental QCLs ${ }^{54}$ due to the asymmetrical form of tunneling into wavevectors above the $\Gamma$ point around resonance; this was neglected here for simplicity and to demonstrate the general approach. Nevertheless, we show in Section IID that our first-order model achieves good agreement with experimental results.

\section{Results}

Experimental $I-V$ characteristics at $77 \mathrm{~K}$ are shown in Fig. 2. The experimental device shows a resonant peak at $0.165 \mathrm{~V}$ with a plateau-like feature between 0.17 and $0.18 \mathrm{~V}$. Previous experimental measurements of AlGaAs RTDs have also observed plateau features in their $I-V$ characteristics. ${ }^{1,55}$ Several theories for their origin have been proposed including intervalley interface scattering ${ }^{56}$ quantized interface states, ${ }^{55,57}$ or time averaged oscillations. ${ }^{58}$ The $I-V$ curves are almost identical on the voltage ramp-up and ramp-down with only a minor current change (shift down of the curve of less than $5 \%$ in current for the ramp-down) and no voltage hysteresis. The shape of the $I-V$ was found to be stable after multiple measurements, as well as after heating the devices to room temperature and subsequent cooling back to cryogenic temperatures performed over a period of several months.

Fig. 3(a) shows the calculated dephasing times over a range of temperatures between states in the emitter reservoir and the ground and first excited states of the quantized well at $V=0.136 \mathrm{~V}$ where the simulations predicted a peak current. The slight discrepancy with the experimentally measured $0.165 \mathrm{~V}$ resonance is attributed to contact resistance effects, as explained later in this section. Dephasing times were found to vary significantly with temperature, decreasing from $94 \mathrm{fs}$ at $6 \mathrm{~K}$ to $33 \mathrm{fs}$ at $300 \mathrm{~K}$ between the quantized emitter state (at $E=-40 \mathrm{meV}$ ) and the ground state in the well. This is due to a significant increase in intrasubband scattering caused mainly by interface roughness and impurity scattering. Dephasing time decreases at higher energies in the emitter reservoir due to the absence of final-state blocking (as they are weakly populated) leading to a faster scattering rate. This absence of final-state blocking causes

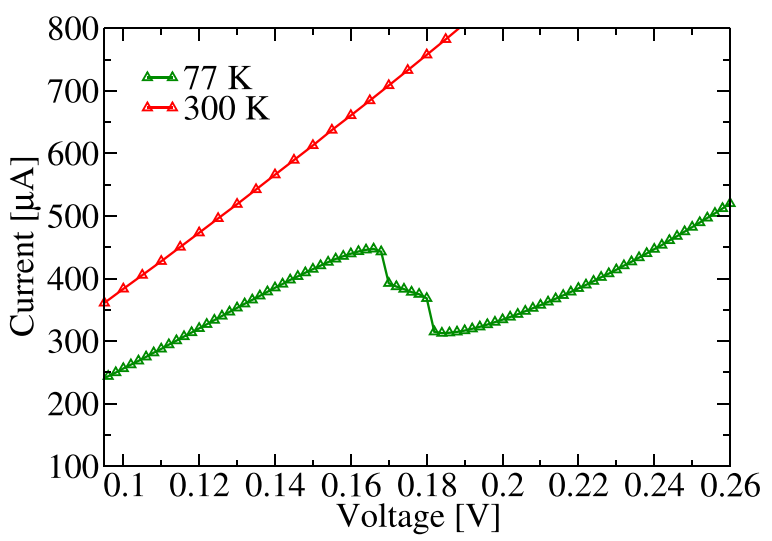

FIG. 2. Experimental $I-V$ characteristics for the $\mathrm{Al}_{0.18} \mathrm{Ga}_{0.82} \mathrm{~N}$ RTD with a $49 \AA$ well and mesa size of $4 \times 4 \mu \mathrm{m}^{2}$ at 77 and $300 \mathrm{~K}$. The positive polarity refers to positive bias applied to the top of the mesa and corresponds to electron injection from the left side in Fig. 1. 

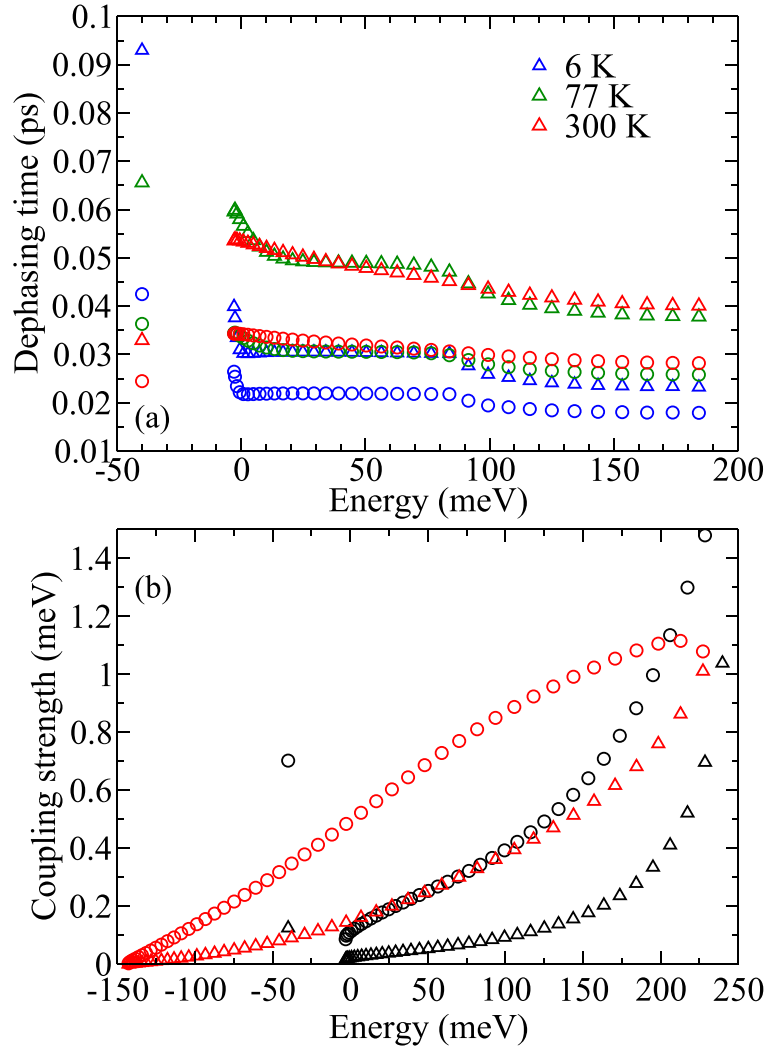

FIG. 3. Calculated dephasing times (a) and coupling strengths (b) for the emitter states for electrons tunneling from the emitter into the ground (triangles) and first excited state (circles) of the RTD well at $0.136 \mathrm{~V}$. A low temperature approximation is used, such that electrons are assumed to initially occupy only states at the bottom of each quantised emitter subband. (b) The coupling strengths between the well and collector states (in red).

the dephasing time for continuum states at $6 \mathrm{~K}$ to be lower than that at higher temperatures. Additionally, the smaller population of the first excited state in the well contributes to a reduction in the dephasing time for tunneling in and out of this state. Initial coupling strengths given by Eq. (4) were found to yield currents larger than the experimentally measured values, and a fitted scaling factor of $37 \%$ was used to account for this overestimation. This is a predictable error since the anti-crossing energy will be overestimated by the tight-binding Hamiltonian. Extraction-coupling strengths were calculated to be larger than emitter-coupling strengths and therefore play a less significant role in determining the vertical electron transport in these devices. Fig. 3(b) shows the calculated coupling strengths for both EW and $\mathrm{CW}$ blocks of the Hamiltonian versus energy. These show that the coupling strength between the quantized emitter state and well states is large due to its localization at the interface. Coupling strengths between the first excited state in the well and the continuum reservoir states are higher due to the reduced confinement of the triangular barrier potential at these higher energies.

Fig. 4 shows the effect of varying IFR parameters on the peak-to-valley (PVR) ratio calculated by the DM model. Interface roughness has been shown to have a significant effect on transport in unipolar devices ${ }^{59}$ and can suppress gain almost completely in tall-barrier QCLs. ${ }^{60}$ Fig. 4 illustrates that increasing the roughness height or correlation length

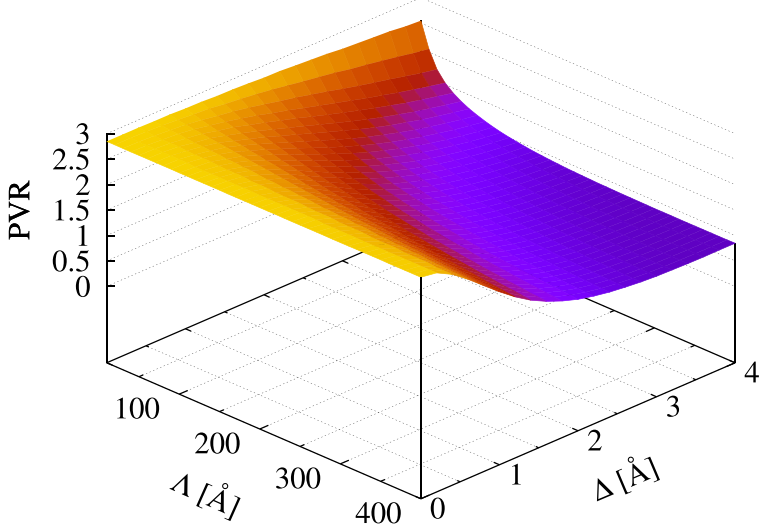

FIG. 4. Peak to valley ratio versus correlation length $(\Lambda)$ and roughness height $(\Delta)$ interface roughness parameters used in dephasing calculation at $77 \mathrm{~K}$.

decreases the PVR by increasing dephasing. Typically, for intersubband scattering, $\Lambda$ in the exponent term of Eq. (8) causes the scattering rate to decrease with increasing $\Lambda$ until it is outweighed by its contribution in the prefactor of Eq. (7), causing scattering to increase after some value. However, since dephasing is the main effect of scattering in RTDs, intrasubband elastic events are of greatest importance. These result in a small change in electron wavevector, and therefore the exponent term in Eq. (8) remains significant at large values of $\Lambda$. Fig. 5 shows the calculated $I-V$ curve from 0.10 to $0.30 \mathrm{~V}$ using interface-roughness parameters $\Delta=2.8 \AA$ and $\Lambda=100 \AA$. Excellent agreement is obtained with the experimentally measured location of the current peak as well as the magnitude of the PVR. These roughness parameters are typical for AlGaAs/GaAs structures such as QCLs ${ }^{61}$ suggesting that interface quality is very high in these MBE grown structures.

The broadening due to dephasing gives improved agreement for the PVR compared with the transfer matrix method which assumes purely ballistic transport. ${ }^{42} \mathrm{~A}$ dephasing time of $0.065 \mathrm{ps}$ at $77 \mathrm{~K}$ for the ground-to-ground state tunneling process results in a full-width at half-maximum (FWHM) broadening of around $10 \mathrm{meV}$ and this increases to $\sim 16 \mathrm{meV}$ for higher energy subbands. Increasing current due to alignment of the first excited state in the well is underestimated

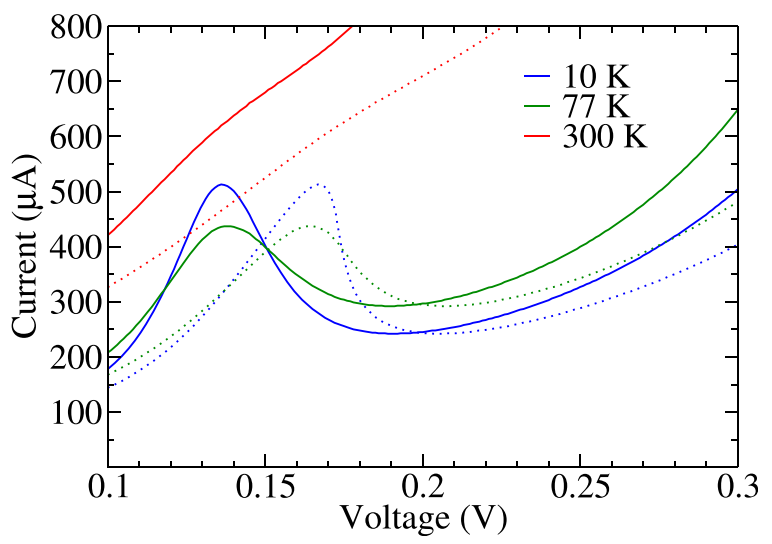

FIG. 5. Simulated current with (dashed) and without (solid) an external series resistance applied to the data. 
by the model (current at the $0.165 \mathrm{~V}$ peak is achieved again at $0.29 \mathrm{~V}$ in the simulation, rather than $0.25 \mathrm{~V}$ observed experimentally) and this is likely due to overestimating the relevant confinement of the excited state in the well compared to the ground state. Our model elucidates that the experimental current peak at $V=0.165 \mathrm{~V}$ arises from the alignment with the quantized emitter state rather than the continuum above the emitter band edge where a combination of lower population, dephasing time, and coupling strength is insufficient to induce an NDR feature. A previous study ${ }^{19}$ of nitride RTDs has also observed alignment features prior to a significant NDR feature and we infer from our model that these can be attributed to alignment with the emitter band-edge in cases where the alignment energies are sufficiently separated. The depth and variance in spatial position of the quantized emitter state are highly sensitive to material parameters which could vary significantly between structures such as contact doping, spacer thickness, and barrier alloy fraction.

It is noteworthy that the position of the NDR is close to that calculated theoretically but lies $29 \mathrm{mV}$ above it. This suggests the presence of a contact resistance, $R_{\mathrm{S}}$, in series with the device that shifts the physical NDR to higher voltages. To estimate the magnitude of the series resistance, we find the $R_{\mathrm{S}}$ as ${ }^{62}$

$$
R_{\mathrm{S}}=\frac{\left(V^{*}-V\right)}{I_{\mathrm{A}}(V)},
$$

where $V$ is the simulated voltage drop across the RTD at resonance, $V^{*}$ is the resonant experimental voltage (including contacts), and $I_{\mathrm{A}}$ is the resonant current. The resulting shifted calculated $I-V$ curves with a $60 \Omega$ contact resistance are shown in Fig. 5; this value is similar to those in Ref. 62. Alternatively, agreement can be achieved by assuming a constant voltage drop due to contacts. However, it is likely a combination of these effects is present.

The absence of a plateau feature in our model is consistent with experimental features being due to timeaveraged oscillations of current when switching between configurations of an empty well while misaligned, and a populated well at resonance. Evidence supporting this is the large well population at resonance $\left(1 \times 10^{11} \mathrm{~cm}^{-2}\right)$ predicted by the model which will induce a Poisson potential acting to push the states out of alignment. It is suggested that a dynamic model which calculates the bandstructure as a function of bias and time would be desirable to investigate this behavior, however it is beyond the scope of this work. It is worth noting that this behavior does not preclude effective resonant tunneling in optoelectronic devices since doping densities per period are much lower in such devices.

\section{E. Validity of the model}

The model presented in this section has been shown to faithfully replicate the disappearance of an NDR feature at high temperatures. Additionally, it allows the effect of changing interface roughness parameters to be calculated conveniently with semi-classical scattering rate calculations. However, the continuum of states used to approximate the emitter and collector reservoirs places an upper limit on the dephasing times where output characteristics will appear physically correct. This is due to the requirement for dephasing to be short enough to broaden the current contribution sufficiently when out of alignment and remove local spikes during instances of exact alignment. The "continuum" state separations are sensitive to the well width used to approximate them, and convergence checks performed indicate that lengths greater than $70 \mathrm{~nm}$ are sufficient. With contact lengths varied between $70 \mathrm{~nm}, 100 \mathrm{~nm}$ (the length used in these simulations), and $200 \mathrm{~nm}$, the change in current and PVR values is negligible.

The model does not account for dynamic changes in state populations that will affect the electric field. This is due to the use of nextnano 3 before the density matrix calculation and therefore we neglect the effect of processes such as dynamic well charge build up. However, this is also the approach used in Refs. 42 and 20 and does not affect conclusions regarding the effect of temperature and IFR parameters on the PVR. Finally, the coupling strength between the well and reservoirs required scaling indicating that this may not be a predictive approach. This is attributed to the large flat potential lying far above the bandedge that will provide significant non-zero contributions in Eq. (4) immediately beyond where the extended bandstructure is replaced. This coupling strength calculation will also lead to unphysical results if weakly bound states with large overlaps between sections are included (such as with the second excited well state if present). This is expected with this type of density matrix approach since it is assumed that all transport is due to tunneling only and requires the "tight-binding" condition to be fulfilled. Therefore, the applicability of this model to devices with very thin layers may be limited. However, a full investigation into the RTD structures where this regime holds is beyond the scope of this article.

\section{SEQUENTIAL TUNNELING DEVICES}

In this section, we compare the theoretical and experimental characteristics of a periodic triple-well structure similar to that in Ref. 24 with a period thickness of $178 \mathrm{~nm}$ in which interface and domain formation effects are not expected to dominate. Ten periods of the structure were grown on a GaN substrate using MBE. The epitaxial layer thicknesses in each period are $\mathbf{2 3} / 47 / \mathbf{1 0} / 23 / \mathbf{2 6} / 49 \AA$ where the $\mathrm{Al}_{0.15} \mathrm{Ga}_{0.85} \mathrm{~N}$ barriers are in bold, the $\mathrm{GaN}$ wells are in regular text, and the underlined well is n-doped with $\mathrm{Si}$ at $1 \times 10^{17} \mathrm{~cm}^{-3}$ to give a sheet density of $5 \times 10^{10} \mathrm{~cm}^{-2}$ per period. Contact layers were $\mathrm{n}^{++}$doped at $2 \times 10^{18} \mathrm{~cm}^{-3}$. The calculated bandstructure of the device at $18.6 \mathrm{kV} / \mathrm{cm}$ is shown in Fig. 6(a), assuming a linear voltage drop across the device. The entire structure is also modeled with the nextnano 3 solver $^{45}$ to check for voltage non-uniformity due to interface accumulation and depletion regions. This is shown in Fig. 6(b), which verifies that the voltage drop is linear across most of the device. 


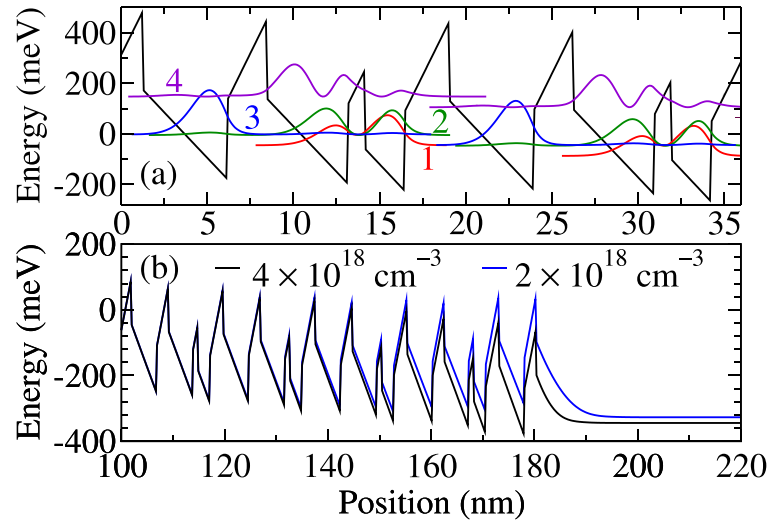

FIG. 6. (a) Bandstructure and wavefunction plot of the sequential tunneling device under an $18.6 \mathrm{kV} / \mathrm{cm}$ bias assuming a linear voltage drop. (b) Trailing few periods of the structure and contact region calculated with nextnano3.

\section{A. Theoretical model}

Eq. (1) is solved in the same way as in Section II, for density matrix terms, which now represent the localized subbands for three sequential periods of the structure ${ }^{63}$

$$
\rho=\left(\begin{array}{ccc}
\rho_{\mathrm{CC}} & \rho_{\mathrm{CU}} & \rho_{\mathrm{CD}} \\
\rho_{\mathrm{UC}} & \rho_{\mathrm{UU}} & 0 \\
\rho_{\mathrm{DC}} & 0 & \rho_{\mathrm{DD}}
\end{array}\right),
$$

where $\mathrm{C}, \mathrm{U}$, and $\mathrm{D}$ refer to the central, upstream, and downstream periods considered, respectively. The Hamiltonian assumes the same form as Eq. (3) with coupling strength energies placed at the interperiod positions. This DM approach assumes translational invariance of the coherence terms so that $\mathrm{CC}$ is equivalent to UU and DD blocks, and the UC (CU) blocks are equivalent to CD (DC) blocks. The anticipated effect of the depletion region band bending is to broaden and reduce the total experimental current because carriers are not resonantly transported for the trailing two periods. All superlattice dopants are assumed to be ionized in the calculations due to the applied field. Interface roughness values identical to those used for RTD simulations were used as both devices were grown in similar conditions. Additionally, the effect of electron-electron interactions is investigated by calculating current both with and without an additional dephasing rate, as was included in Section II.

\section{B. Results}

The experimental and calculated current are shown in Fig. 7(a) as a function of the applied electric field, along with the subband energy variation. Two strong alignment features are apparent in the simulated current. From our theoretical model, it is deduced that these arise from the ground state of the $49 \AA$ well coming into resonance with the upstream states and downstream states at different biases. This behavior is less readily apparent in the experimental data, since the sequential tunneling features are obscured by a large parasitic current, which is likely due to traps and other current paths associated with defects such as screw dislocations. ${ }^{64}$ However, the alignment features are clearly visible as plateaus in the differential resistance and there is

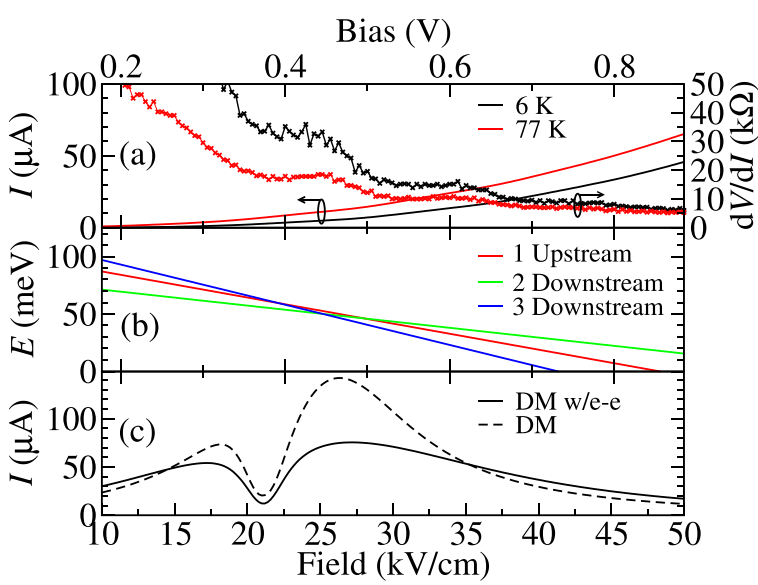

FIG. 7. (a) Experimental current and differential resistance. The positive polarity refers to positive bias applied to the top of the mesa and corresponds to electron injection from the left side in Fig. 6. (b) Calculated subband alignment energies at $77 \mathrm{~K}$. (c) Current calculated with the density matrix formalism with and without additional e-e dephasing at $77 \mathrm{~K}$.

excellent agreement between the experimental and simulated alignment voltages, indicating that effects of electric field domain formation are negligible on overall current. The $I-V$ features are reproducible and do not show any evidence of hysteresis within the error of the measurements.

Fig. 6(b) shows that by doubling the contact doping, nextnano3 predicts that residual bending near the end of the device can be suppressed by increased screening due to the ionized dopants. While interface charge effects do not have a significant effect on sequential tunneling in the majority of the device, careful control of the doping and spacer layers is necessary for the most efficient overall electron transport and simulations suggest that contact doping should generally be as high as possible.

The effect of electron-electron scattering is to reduce and broaden the vertical electron transport as shown in Fig. 7(c) and this must be taken into account in superlattice doping considerations for optimized structures. Simulations of the device at $6 \mathrm{~K}$ resulted in negligible $I-V$ differences compared with simulations at $77 \mathrm{~K}$. This was unexpected since the experimental data show a shift to lower resistance at higher temperatures. This discrepancy can be explained by the low lattice temperatures at which phonon scattering is insignificant, thus causing simulations to be similar at both temperatures. The experimental decrease in resistance is then consistent with recent studies on the thermal activation of charge traps ${ }^{65}$ and resembles features of Frenkel-Poole tunneling. ${ }^{37}$

Frenkel-Poole tunneling enhances current flow with a linear dependence between the current divided by the electric field and the square root of the electric field. Fig. 8 shows a clear linear dependence between these functions however the linear electric field over the active region could not be used to fit Frenkel-Poole or phonon emission expressions typically applied to HEMTs. ${ }^{66}$ This may indicate that the electric field relevant for these expressions is a complex interaction between forward applied bias, reverse barrier fields, and domain formation effects (if present), or that the leakage current comes from another mechanism entirely. Along with 


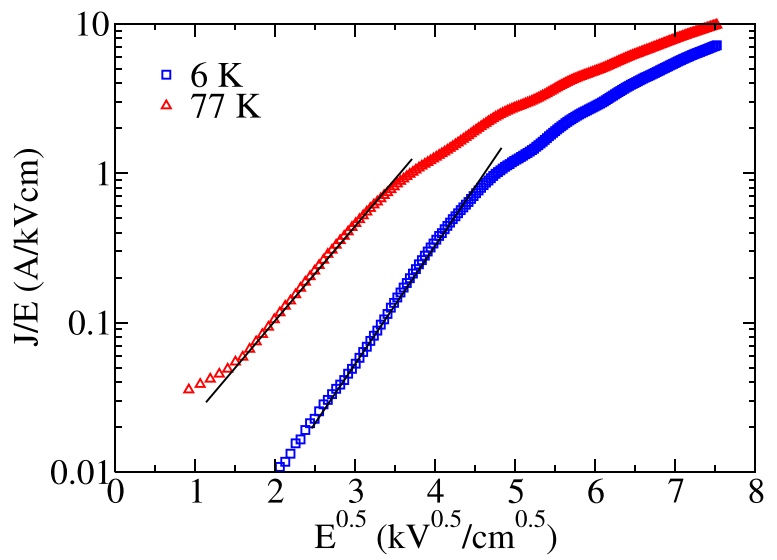

FIG. 8. Experimental sequential tunneling current divided by electric field vs square root of electric field at $6 \mathrm{~K}$ and $77 \mathrm{~K}$ along with straight line fits.

previous studies on the electron charge trapping, these results indicate that room temperature sequential tunneling is feasible provided material quality and suppression of defects is improved further. This is important for thicker structures, such as QCLs, which require up to $10-\mu \mathrm{m}$-thick active regions, although several studies have been performed to minimize strain with balanced substrates. ${ }^{67,68}$

\section{CONCLUSIONS}

In summary, we have studied the vertical electron transport in different types of $\mathrm{AlGaN} / \mathrm{GaN}$ heterostructures both theoretically and experimentally. Excellent agreement has been obtained between measured current-voltage characteristics and values calculated by the density matrix formalism. This is a general approach, which can also be applied to QCL devices engineered to give optical gain, and is therefore a useful design and optimization tool. Fitted interface roughness values indicate high interface quality however sequential tunneling devices were observed to have a significant parallel parasitic current, likely due to defects. Our results indicate the feasibility of quantum devices such as QCLs provided defect density is reduced further and interface roughness is kept low.

\section{ACKNOWLEDGMENTS}

The authors are grateful to EPSRC (UK) for a DTG award and to the University of Leeds FIRC 2011 grant. Experimental work was supported by the NSF Award No. ECCS-1001431 and from the Defense Advanced Research Project Agency (DARPA) under Contract No. D11PC20027.

${ }^{1}$ R. Köhler, A. Tredicucci, F. Beltram, H. E. Beere, E. H. Linfield, A. G. Davies, D. A. Ritchie, R. C. Iotti, and F. Rossi, Nature 417, 156 (2002).

${ }^{2}$ B. S. Williams, Nat. Photonics 1, 517 (2007).

${ }^{3}$ L. Li, L. Chen, J. Zhu, J. Freeman, P. Dean, A. Valavanis, A. Davies, and E. Linfield, Electron. Lett 50, 309 (2014).

${ }^{4}$ S. Fathololoumi, E. Dupont, C. Chan, Z. Wasilewski, S. Laframboise, D. Ban, A. Mátyás, C. Jirauschek, Q. Hu, and H. C. Liu, Opt. Express 20, 3866 (2012).

${ }^{5}$ A. W. M. Lee, Q. Qin, S. Kumar, B. S. Williams, Q. Hu, and J. L. Reno, Appl. Phys. Lett. 89, 141125 (2006).
${ }^{6}$ V. D. Jovanović, D. Indjin, Z. Ikonić, and P. Harrison, Appl. Phys. Lett. 84, 2995 (2004).

${ }^{7}$ H. Yasuda, T. Kubis, I. Hosako, and K. Hirakawa, J. Appl. Phys. 111, 083105 (2012).

${ }^{8}$ W. Freeman and G. Karunasiri, Appl. Phys. Lett. 102, 152111 (2013).

${ }^{9}$ B. Levine, J. Appl. Phys. 74, R1 (1993).

${ }^{10}$ R. Tsu and L. Esaki, Appl. Phys. Lett. 22, 562 (1973).

${ }^{11}$ R. C. Bowen, G. Klimeck, R. K. Lake, W. R. Frensley, and T. Moise, J. Appl. Phys. 81, 3207 (1997).

${ }^{12}$ B. R. Bennett, R. Magno, J. B. Boos, W. Kruppa, and M. G. Ancona, Solid-State Electron. 49, 1875 (2005)

${ }^{13}$ S. Golka, C. Pflügl, W. Schrenk, G. Strasser, C. Skierbiszewski, M. Siekacz, I. Grzegory, and S. Porowski, Appl. Phys. Lett. 88, 172106 (2006).

${ }^{14}$ E. Baumann, F. R. Giorgetta, D. Hofstetter, H. Wu, W. J. Schaff, L. F. Eastman, and L. Kirste, Appl. Phys. Lett. 86, 032110 (2005).

${ }^{15}$ C. Bayram, Z. Vashaei, and M. Razeghi, Appl. Phys. Lett. 96, 042103 (2010).

${ }^{16}$ Z. Vashaei, C. Bayram, and M. Razeghi, J. Appl. Phys. 107, 083505 (2010).

${ }^{17}$ C. Bayram, Z. Vashaei, and M. Razeghi, Appl. Phys. Lett. 97, 181109 (2010).

${ }^{18}$ Y. Shao, S. D. Carnevale, A. T. M. G. Sarwar, R. C. Myers, and W. Lu, J. Vac. Sci. Technol., B 31, 06FA03 (2013).

${ }^{19}$ D. Li, L. Tang, C. Edmunds, J. Shao, G. Gardner, M. J. Manfra, and O. Malis, Appl. Phys. Lett. 100, 252105 (2012).

${ }^{20} \mathrm{D}$. Li, J. Shao, L. Tang, C. Edmunds, G. Gardner, M. J. Manfra, and O. Malis, Semicond. Sci. Technol. 28, 074024 (2013).

${ }^{21}$ M. Boucherit, A. Soltani, E. Monroy, M. Rousseau, D. Deresmes, M. Berthe, C. Durand, and J.-C. De Jaeger, Appl. Phys. Lett. 99, 182109 (2011).

${ }^{22}$ A. Kikuchi, R. Bannai, K. Kishino, C.-M. Lee, and J.-I. Chyi, Appl. Phys. Lett. 81, 1729 (2002).

${ }^{23}$ L. Yang, H. He, W. Mao, and Y. Hao, Appl. Phys. Lett. 99, 153501 (2011).

${ }^{24}$ F. F. Sudradjat, W. Zhang, K. Driscoll, Y. Liao, A. Bhattacharyya, C. Thomidis, L. Zhou, D. J. Smith, T. D. Moustakas, and R. Paiella, Phys. Status Solidi C 9, 588 (2012).

${ }^{25}$ S. Sakr, Y. Kotsar, M. Tchernycheva, E. Warde, N. Isac, E. Monroy, and F. H. Julien, Appl. Phys. Express 5, 052203 (2012).

${ }^{26}$ R. Songmuang, G. Katsaros, E. Monroy, P. Spathis, C. Bougerol, M. Mongillo, and S. D. Franceschi, Nano Lett. 10, 3545 (2010).

${ }^{27}$ L. Rigutti, G. Jacopin, A. D. L. Bugallo, M. Tchernycheva, E. Warde, F. H. Julien, R. Songmuang, E. Galopin, L. Largeau, and J.-C. Harmand, Nanotechnology 21, 425206 (2010).

${ }^{28}$ E. Li, Z. Cui, M. Liu, and X. Wang, Integr. Ferroelectr. 137, 134 (2012).

${ }^{29}$ S. D. Carnevale, C. Marginean, P. J. Phillips, T. F. Kent, A. T. M. G. Sarwar, M. J. Mills, and R. C. Myers, Appl. Phys. Lett. 100, 142115 (2012).

${ }^{30}$ C. Gmachl, H. M. Ng, S.-N. George Chu, and A. Y. Cho, Appl. Phys. Lett. 77, 3722 (2000).

${ }^{31}$ M. Tchernycheva, L. Nevou, L. Doyennette, F. H. Julien, E. Warde, F. Guillot, E. Monroy, E. Bellet-Amalric, T. Remmele, and M. Albrecht, Phys. Rev. B 73, 125347 (2006).

${ }^{32}$ C. Edmunds, L. Tang, M. Cervantes, M. Shirazi-HD, J. Shao, A. Grier, A. Valavanis, J. D. Cooper, D. Li, G. Gardner, D. N. Zakharov, Z. Ikonić, D. Indjin, P. Harrison, M. J. Manfra, and O. Malis, Phys. Rev. B 88, 235306 (2013).

${ }^{33}$ H. Machhadani, Y. Kotsar, S. Sakr, M. Tchernycheva, R. Colombelli, J. Mangeney, E. Bellet-Amalric, E. Sarigiannidou, E. Monroy, and F. H. Julien, Appl. Phys. Lett. 97, 191101 (2010).

${ }^{34}$ D. Hofstetter, D. P. Bour, and L. Kirste, Appl. Phys. Lett. 104, 241107 (2014).

${ }^{35}$ W. Terashima and H. Hirayama, Phys. Status Solidi C 8, 2302 (2011).

${ }^{36}$ F. Sudradjat, W. Zhang, K. Driscoll, Y. Liao, A. Bhattacharyya, C. Thomidis, L. Zhou, D. J. Smith, T. D. Moustakas, and R. Paiella, J. Appl. Phys. 108, 103704 (2010).

${ }^{37}$ O. Mitrofanov and M. Manfra, J. Appl. Phys. 95, 6414 (2004).

${ }^{38}$ D. N. Nath, Z. C. Yang, C.-Y. Lee, P. S. Park, Y.-R. Wu, and S. Rajan, Appl. Phys. Lett. 103, 022102 (2013).

${ }^{39}$ B. A. Biegel and J. D. Plummer, Phys. Rev. B 54, 8070 (1996).

${ }^{40}$ F. Rossi, P. Poli, and C. Jacoboni, Semicond. Sci. Technol. 7, 1017 (1992).

${ }^{41}$ K. L. Jensen and F. A. Buot, Phys. Rev. Lett. 66, 1078 (1991).

${ }^{42}$ S. Sakr, E. Warde, M. Tchernycheva, and F. H. Julien, J. Appl. Phys. 109, 023717 (2011). 
${ }^{43}$ M. Boucherit, A. Soltani, M. Rousseau, J.-L. Farvacque, and J.-C. DeJaeger, J. Appl. Phys. 112, 114305 (2012).

${ }^{44}$ G. Beji, Z. Ikonić, C. A. Evans, D. Indjin, and P. Harrison, J. Appl. Phys. 109, 013111 (2011).

${ }^{45}$ S. Birner, T. Zibold, T. Andlauer, T. Kubis, M. Sabathil, A. Trellakis, and P. Vogl, IEEE Trans. Electron Devices 54, 2137 (2007).

${ }^{46}$ J. D. Cooper, A. Valavanis, Z. Ikonić, P. Harrison, and J. E. Cunningham, J. Appl. Phys. 108, 113109 (2010).

${ }^{47}$ A. Yariv, C. Lindsey, and U. Sivan, J. Appl. Phys. 58, 3669 (1985).

${ }^{48}$ M. A. Talukder and C. R. Menyuk, New J. Phys. 13, 083027 (2011).

${ }^{49}$ H. Callebaut and Q. Hu, J. Appl. Phys. 98, 104505 (2005).

${ }^{50}$ C. Weber, A. Wacker, and A. Knorr, Phys. Rev. B 79, 165322 (2009).

${ }^{51}$ A. Valavanis, Z. Ikonić, and R. W. Kelsall, Phys. Rev. B 77, 075312 (2008).

${ }^{52}$ C. Sanderson, "Armadillo: An open source $\mathrm{C}++$ linear algebra library for fast prototyping and computationally intensive experiments," Technical Report (NICTA, Australia, 2010).

${ }^{53}$ E. Anderson, Z. Bai, J. Dongarra, A. Greenbaum, A. McKenney, J. Du Croz, S. Hammerling, J. Demmel, C. Bischof, and D. Sorensen, in Proceedings of the 1990 ACM/IEEE Conference on Supercomputing, Supercomputing'90 (IEEE Computer Society Press, Los Alamitos, CA, USA, 1990), pp. 2-11.

${ }^{54}$ R. Terazzi, T. Gresch, A. Wittmann, and J. Faist, Phys. Rev. B 78, 155328 (2008).

${ }^{55}$ A. Fediai and V. Moskaliuk, in Electronics and Nanotechnology (ELNANO), 2013 IEEE XXXIII International Scientific Conference, Ukraine (2013), pp. 107-111.
${ }^{56}$ I. Abramov, N. Kolomeitseva, and I. Romanova, Russ. Microlectron. 41, 314 (2012).

${ }^{57}$ P. Zhao, H. L. Cui, D. Woolard, K. L. Jensen, and F. A. Buot, J. Appl. Phys. 87, 1337 (2000)

${ }^{58}$ A. Sakurai and Y. Tanimura, J. Phys. Soc. Jpn. 82, 033707 (2013).

${ }^{59}$ J. B. Khurgin, Y. Dikmelik, P. Q. Liu, A. J. Hoffman, M. D. Escarra, K. J. Franz, and C. F. Gmachl, Appl. Phys. Lett. 94, 091101 (2009).

${ }^{60}$ C. W. I. Chan, Q. Hu, and J. L. Reno, Appl. Phys. Lett. 103, 151117 (2013).

${ }^{61}$ E. Dupont, S. Fathololoumi, Z. R. Wasilewski, G. Aers, S. R. Laframboise, M. Lindskog, S. G. Razavipour, A. Wacker, D. Ban, and H. C. Liu, J. Appl. Phys. 111, 073111 (2012).

${ }^{62}$ Y. Hou, W.-P. Wang, N. Li, W. Lu, and Y. Fu, J. Appl. Phys. 104, 074508 (2008).

${ }^{63}$ T. V. Dinh, A. Valavanis, L. J. M. Lever, Z. Ikonić, and R. W. Kelsall, Phys. Rev. B 85, 235427 (2012).

${ }^{64}$ S. J. Pearton, J. C. Zolper, R. J. Shul, and F. Ren, J. Appl. Phys. 86, 1 (1999).

${ }^{65}$ S. Sakr, E. Warde, M. Tchernycheva, L. Rigutti, N. Isac, and F. H. Julien, Appl. Phys. Lett. 99, 142103 (2011).

${ }^{66}$ A. M. Katzenmeyer, F. Léonard, A. A. Talin, P.-S. Wong, and D. L. Huffaker, Nano Lett. 10, 4935 (2010).

${ }^{67}$ K. Berland, T. G. Andersson, and P. Hyldgaard, Phys. Rev. B 84, 245313 (2011).

${ }^{68}$ V. Jovanović, Z. Ikonić, D. Indjin, P. Harrison, V. Milanović, and R. Soref, J. Appl. Phys. 93, 3194 (2003). 